\title{
THE ALBANESE MAPPING FOR A PUNCTUAL HILBERT SCHEME: I. IRREDUCIBILITY OF THE FIBERS
}

BY

\author{
MARK E. HUIBREGTSE
}

\begin{abstract}
Let $f: X \rightarrow A$ be the canonical mapping from an algebraic surface $X$ to its Albanese variety $A, X(n)$ the $n$-fold symmetric product of $X$, and $H_{X}^{n}$ the punctual Hilbert scheme parameterizing 0 -dimensional closed subschemes of length $n$ on $X$. The latter is a nonsingular and irreducible variety of dimension $2 n$, and the "Hilbert-Chow" morphism $\sigma_{n}: H_{X}^{n} \rightarrow X(n)$ is a birational map which desingularizes $X(n)$.

This paper studies the composite morphism

$$
\varphi_{n}: H_{X}^{n} \stackrel{\sigma_{n}}{\rightarrow} X(n) \stackrel{f_{n}}{\rightarrow} A,
$$

where $f_{n}$ is obtained from $f$ by addition on $A$. The main result (Part 1 of the paper) is that for $n \gg 0$, all the fibers of $\varphi_{n}$ are irreducible and of dimension $2 n-q$, where $q=\operatorname{dim} A$. An interesting special case (Part 2 of the paper) arises when $X=A$ is an abelian surface; in this case we show (for example) that the fibers of $\varphi_{n}$ are nonsingular, provided $n$ is prime to the characteristic.
\end{abstract}

Introduction. Let $X$ be an irreducible and nonsingular projective variety of dimension $d>0$, defined over an algebraically closed ground field. Let $f$ : $X \rightarrow A$ be the canonical map from $X$ to its Albanese variety $A$; over the complex numbers this is the complex torus $C^{q} / L$, where $L$ is the lattice generated by the $2 q$ periods of a basis of holomorphic 1-forms on $X$. If $X(n)$ is the $n$-fold symmetric product of $X$, whose points represent the 0 -cycles on $X$ of degree $n$, then the map $f: X \rightarrow A$ induces by addition on $A$ a map,

$$
f_{n}: X(n) \rightarrow A,
$$

which is in fact the Albanese mapping of $X(n)$.

In case $X$ is a curve $C$, the Albanese variety is the Jacobian $J$ of $C$, and the fibers of $f_{n}: C(n) \rightarrow J$ are projective spaces which represent the complete linear systems of degree $n$ on $C$ (the Abel-Jacobi theorem). The classical Riemann-Roch theorem computes the dimensions of these fibers for all $n>0$. In particular, Riemann's theorem is the assertion that for $n \gg 0$, all of the fibers of $f_{n}: C(n) \rightarrow J$ have the same dimension $n-g$, where $g$ is the genus of $C$.

Received by the editors November 4, 1976.

AMS (MOS) subject classifications (1970). Primary 14C05; Secondary 14K99, 14E99.

Key words and phrases. Punctual Hilbert scheme, symmetric product, Albanese variety, Albanese mapping, algebraic surface. 
It is known that the natural generalization of Riemann's theorem to the mapping $f_{n}: X(n) \rightarrow A$ is true where $\operatorname{dim} X=d>1$. That is, for $n \gg 0$, all of the fibers of $f_{n}$ are irreducible and of the same dimension $d n-q$, where $q=\operatorname{dim} A[10]$. In seeking further generalizations (for example, analogues of the full Riemann-Roch theorem) one confronts the fact that $X(n)$ has singularities when $\operatorname{dim} X>1$, a "defect" not shared by the nonsingular variety $C(n)$. Consequently the fibers of $f_{n}$ can, and do, acquire singularities which can seriously interfere.

If $X$ is a surface, however, there is available a natural desingularization of $X(n)$. This is the Hilbert scheme $H_{X}^{n}$, an irreducible and nonsingular projective variety of dimension $2 n$ whose points represent the closed subschemes of $X$ having Hilbert polynomial $n$. With each such subscheme $Z$ there is naturally associated a 0 -cycle of degree $n$ (a weighted sum of the points in the support of $Z$ ); this gives a birational map

$$
\sigma_{n}: H_{X}^{n} \rightarrow X(n)
$$

which is an isomorphism over the smooth locus of $X(n)$, whose points represent the 0 -cycles $x_{1}+\cdots+x_{n}$ with $n$ distinct summands. (All these results may be found in [2]. Morphisms analogous to $\sigma_{n}$ exist for $X$ of any dimension $d>0$, but $H_{X}^{n}$ is not in general irreducible for $d>2$ [6]. If $d=1$, $\left.H_{C}^{n}=C(n)[7].\right)$

The main purpose of this paper is to prove that when $X$ is a surface and $n \gg 0$, all of the fibers of the composite mapping

$$
\varphi_{n}: H_{X}^{n} \stackrel{\sigma_{n}}{\rightarrow} X(n) \stackrel{f_{n}}{\rightarrow} A
$$

are irreducible and of the same dimension (Corollary 1 of Theorem 3 ). The proof of this result is the focus of Part 1 of the paper. The necessary preliminaries include a detailed study of the map $f_{n}$ restricted to the singular locus of $X(n)$, which is carried out for $X$ of any dimension.

Part 2 of the paper treats the special case where $X$ is taken to be an abelian variety $A$, that is, $X$ equals its own Albanese variety. The principal results of Part 1 (those that follow Theorem 3) are shown to hold for all $n>0$, rather than just for $n \gg 0$. Moreover, additional information is obtained, notably Corollary 1 of Theorem 6, which states that if $A$ is an abelian surface and $n$ is prime to the characteristic, then all fibers of the mapping $\varphi_{n}: H_{A}^{n} \rightarrow A$ are nonsingular varieties which desingularize the fibers of $f_{n}: A(n) \rightarrow A$. The arguments in Part 2 are based on the group structure of $A$, and are essentially independent of Part 1.

A forthcoming paper will study the question of smoothness for the mapping $\varphi_{n}: H_{X}^{n} \rightarrow A$, when $X$ is a surface; it can be viewed as a step in the direction of finding a "Roch" theorem to complement the "Riemann" theorem proved here. It should be noted, however, that unlike the case of curves, 
certain fibers of $\varphi_{n}$ can be singular, even in characteristic 0 and for arbitrarily large $n$; examples will be given.

The author wishes to thank Professors Arthur Mattuck and Steven Kleiman for all their help.

\section{PART 1}

Remark on terminology. In addition to those established in the introduction, we make the following conventions. A variety is a reduced, separated, not necessarily irreducible scheme of finite type over a field $k$, the latter usually taken to be algebraically closed. By a point of $V$ we mean a geometric point, i.e., a map Spec $K \rightarrow V$ where $K$ is an algebraically closed extension of the ground field. If $V$ is irreducible, a generic point of $V$ is a point localized at the scheme-theoretic generic point of $V$, and a set $\left\{v_{1}, \ldots, v_{n}\right\}$ of points of $V$ is a set of independent generic points if $\left(v_{1}, \ldots, v_{n}\right)$ is a generic point of the product $V^{n}$.

The fibers of a morphism of varieties are taken to be varieties, i.e., they are always given their reduced structure. The generic fiber refers to the fiber over a generic point as understood above. (In Part 2 we do not need the language of generic points; there, a point is a rational point over the (algebraically closed) ground field.)

Finally, if $v_{1}+\cdots+v_{n}$ is a 0 -cycle on $V$, the associated point of $V(n)$ is written $\left(v_{1}+\cdots+v_{n}\right)$.

1. The varieties $X(\pi)$. Until further notice, let $X$ be a projective variety of dimension $d>0$, irreducible and nonsingular, and let $f: X \rightarrow A$ be its Albanese mapping. We assume throughout that $\operatorname{dim} A=q>0$. Of particular interest for us is the nonsmooth locus of $X(n)$ and the behavior of the map $f_{n}$ : $X(n) \rightarrow A$ restricted to the nonsmooth locus. If $\left(x_{1}+\cdots+x_{n}\right) \in X(n)$, let $r<n$ be the number of distinct $x_{i}$. Then, as noted above, $\left(x_{1}+\cdots+x_{n}\right)$ is a smooth point of $X(n)$ if and only if $r=n$. We call $r$ the size of $\left(x_{1}\right.$ $\left.+\cdots+x_{n}\right)$.

Let $\pi=\left(n_{1}, \ldots, n_{r}\right)$ be a partition of $n$ into $r$ parts, which means each $n_{i}>0$ and $\sum_{i=1}^{r} n_{i}=n$. We call $r$ the size of $\pi$ and $n$, following standard usage, the weight of $\pi$.

To every partition $\pi=\left(n_{1}, \ldots, n_{r}\right)$ of weight $n$ and size $r$ there corresponds a closed irreducible subvariety $X(\pi)$ of $X(n)$. By definition, $X(\pi)$ is the image of the composite morphism

$$
\theta_{\pi}: X^{r^{\Delta n_{1} \times \cdots \times \Delta n_{r}}} X^{n_{1}} \times \cdots \times X^{n_{r}}=X^{n} \stackrel{\theta_{n}}{\rightarrow} X(n),
$$

where $\Delta n_{i}: X \rightarrow X^{n_{i}}$ is the diagonal map and $\theta_{n}$ is the quotient map (which sends $\left(x_{1}, \ldots, x_{n}\right)$ to $\left.\left(x_{1}+\cdots+x_{n}\right)\right)$. Thus $\theta_{\pi}\left(x_{1}, \ldots, x_{r}\right)=\left(\sum_{i=1}^{r} n_{i} x_{i}\right)$. Since $X^{r}$ is complete and irreducible, it follows that its image $X(\pi)$ is a closed irreducible subvariety of $X(n)$. 
The following are immediate consequences of the definition:

(2) If $\left\{x_{1}, \ldots, x_{r}\right\}$ is a set of independent generic points of $X$, then $\left(\sum_{i=1}^{r} n_{i} x_{i}\right)$ is a generic point of $X(\pi)$.

(3) The points of $X(\pi)$ represent the 0 -cycles of the form $\sum_{i=1}^{r} n_{i} x_{i}$, where not all the $x_{i}$ need be distinct.

(4) $\operatorname{dim} X(\pi)=d r$, where $d=\operatorname{dim} X$ and $r$ is the size of $\pi$.

We write $f_{\pi}: X(\pi) \rightarrow A$ for the restriction to $X(\pi)$ of the morphism $f_{n}$ : $X(n) \rightarrow A$. (If, as is standard, we let $n$ represent the partition $(1, \ldots, 1)$ of weight and size $n$, then $X(n), \theta_{n}$ and $f_{n}$ are exactly $X(\pi), \theta_{\pi}$, and $f_{\pi}$ for $\pi=n$, so the notation is consistent.)

The next two sections study the map $f_{\pi}$. In $\$ 2$ we show that $f_{\pi}$ is surjective if the size of $\pi$ is sufficiently large, and in $\$ 3$ we consider the fibers of $f_{\pi}$ (dimension and irreducibility).

2. Surjectivity of $f_{\pi}$. Let $B$ be an abelian variety and $Z, Z^{\prime}$ subvarieties of $B$. We write $Z+Z^{\prime}$ for the image of the map

$$
Z \times Z^{\prime} \rightarrow B \times B \stackrel{+}{\rightarrow} B
$$

the sum $Z+\cdots+Z$ ( $m$ summands) is abbreviated $m Z$. We say $Z$ generates $B$ if the inclusion $m Z \hookrightarrow B$ dominates $B$ for $m \gg 0$. As usual we write $m \delta$ : $B \rightarrow B$ for the isogeny of multiplication by $m$ on $B$. Then $m \delta(Z)$ is the image of $Z$ under $m \delta$ and should not be confused with $m Z$.

It is clear that

(5) if $Z$ and $Z^{\prime}$ are closed (resp. irreducible) subvarieties of $B$, then so are $Z+Z^{\prime}, m Z$, and $m \delta(Z)$,

since each of these is the image of a complete (resp. irreducible) variety.

Proposition 1. Let $B$ be an abelian variety of dimension $p>0$. Let $Z_{1}, \ldots Z_{s}$ be closed irreducible subvarieties of $B$ each of which generates $B$, with, say, $\operatorname{dim} Z_{1}>\operatorname{dim} Z_{i}$ for $1<i<s$. Then for $s>p-\operatorname{dim} Z_{1}$, and all $n_{i}>0$,

$$
n_{1} \delta\left(Z_{1}\right)+\cdots+n_{s} \delta\left(Z_{s}\right)=B .
$$

To prove this, we use two lemmas.

LEMMA 1. If $Z$ is an irreducible subvariety of $B$ that generates $B$, then so is $n \delta(Z)$ for any $n>0$.

Proof. We have that $n \delta(Z)$ is irreducible by (5). To see $n \delta(Z)$ generates $B$ consider the commutative diagram ( $m$ factors)

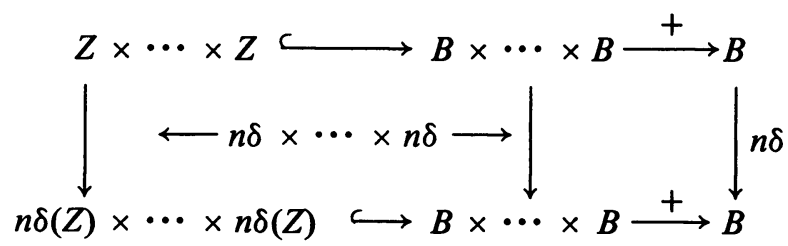


where $m$ is chosen so that the top row is dominating. Since the rightmost vertical arrow is also dominating, it follows that the bottom row is dominating, whence the lemma.

LEMMA 2. Let $W$ be a proper irreducible subvariety of $B$, and $Z$ an irreducible subvariety of $B$ that generates $B$. Then $\operatorname{dim} W<\operatorname{dim}(Z+W)$.

Proof. Clearly we may assume $Z$ contains the identity element of $B$, so $W \subseteq Z+W$ and $\operatorname{dim}(W) \leqslant \operatorname{dim}(Z+W)$. In addition, since $\operatorname{dim}(W)=$ $\operatorname{dim}(\bar{W})$ and (as is easily checked)

$$
\operatorname{dim}(Z+W)=\operatorname{dim}(\bar{Z}+\bar{W}),
$$

we may assume $Z$ and $W$ are closed. Thus $Z+W$ is closed and irreducible (5), and the equality $\operatorname{dim}(W)=\operatorname{dim}(Z+W)$ forces $W=Z+W$, hence

$$
W=Z+W=2 Z+W=\cdots=m Z+W \text {. }
$$

But $m Z=B$ for $m \gg 0$, since $Z$ generates $B$, so $W=B+W=B$, a contradiction.

Proof of Proposition 1. Since $\operatorname{dim} Z_{i}=\operatorname{dim} n_{i} \delta\left(Z_{i}\right)$ for $1<i<s$, Lemma 1 reduces us to proving $Z_{1}+\cdots+Z_{s}=B$. If $Z_{1}+\cdots+Z_{s} \neq B$, by using (5) and Lemma 2 we see

$\operatorname{dim} B>\operatorname{dim}\left(Z_{1}+\cdots+Z_{s}\right)>\operatorname{dim}\left(Z_{1}+\cdots+Z_{s-1}\right)>\cdots>\operatorname{dim} Z_{1}$. Since the dimension falls by at least one in each of the $s$ steps to $Z_{1}$, it follows that

$$
\operatorname{dim} B=p>\operatorname{dim} Z_{1}+s,
$$

contradicting the choice of $s$.

Proposition 2. Let $\pi=\left(n_{1}, \ldots, n_{r}\right)$ be any partition of size $r>q-$ $\operatorname{dim} f(X)$, where $q=\operatorname{dim} A$. Then $f_{\pi}: X(\pi) \rightarrow A$ is surjective.

Proof. Since $X$ is an irreducible projective variety, $f(X)$ is a closed irreducible subvariety of $A$ that generates $A$. Keeping in mind (3), it is easy to check that

$$
\begin{aligned}
f_{\pi}(X(\pi)) & =n_{1} \delta(f(X))+\cdots+n_{r} \delta(f(X)) \\
& =A, \text { by Proposition } 1 .
\end{aligned}
$$

3. Dimension and irreducibility of the fibers of $f_{\pi}$. The following results are proved in [10].

(6) If $n \gg 0$, the generic fiber of $f_{n}: X(n) \rightarrow A$ is (nonempty and) irreducible. (It suffices to take $n>g$, where $g$ is the genus of a generic 1-dimensional linear section on $X$.)

(7) Let $n_{0}$ be the least $n>0$ for which the conclusion of (6) holds. If $n>n_{0}+q$, then every fiber of $f_{n}$ is irreducible and of dimension $d n-q$.

Theorems 1 and 2 below extend these results to the mapping $f_{\pi}: X(\pi) \rightarrow A$. 
Theorem 1 (in case $X$ is a surface) plays an important role in the proof of Theorem 3, our main goal. Theorem 2 has no application in this paper, but is included for completeness.

For the proofs of Theorems 1 and 2 we need

LEMMA 3. Let $Y_{1}, Y_{2}$ and $Z$ be three irreducible varieties of dimensions $d_{1}, d_{2}$, and $s$, respectively, and let $G_{1}: Y_{1} \rightarrow Z, G_{2}: Y_{2} \rightarrow Z$ be morphisms, where $G_{1}$ is assumed dominating. Form the cartesian diagram:

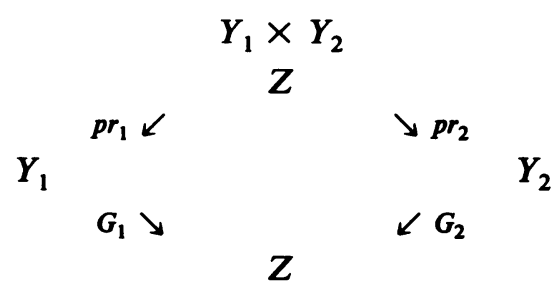

(a) Let $V$ be an irreducible subvariety of $Y_{1} \times_{Z} Y_{2}$, which dominates $Y_{1}$ by the first projection, and satisfies $\operatorname{dim} V>d_{1}+d_{2}-s$. Then

(i) $\operatorname{dim} V=d_{1}+d_{2}-s$,

(ii) $V$ dominates $Y_{2}$ by the second projection, and

(iii) $\bar{V}$ (the closure of $V$ ) is an irreducible component of $Y_{1} \times_{Z} Y_{2}$.

(b) If $G_{2}$ is assumed dominating and $G_{1}$ has irreducible generic fiber, then there exists a unique irreducible component $W$ of $Y_{1} \times_{Z} Y_{2}$ that dominates $Y_{2}$ by the second projection, and $\operatorname{dim} W=d_{1}+d_{2}-s$.

Proof. (a) Let $v=\left(v_{1}, v_{2}\right)$ be a generic point of $V$, where $v_{i} \in Y_{i}, i=1,2$; by hypothesis $v_{1}$ is a generic point of $Y_{1}$ and $G_{1}\left(v_{1}\right)=z=G_{2}\left(v_{2}\right)$ is a generic point of $Z$. Note that both residue fields $k\left(v_{1}\right)$ and $k\left(v_{2}\right)$ contain $k(z)$, and that $\operatorname{dtr}_{k} k\left(v_{1}\right)=d_{1}, \operatorname{dtr}_{k} k(z)=s$, and $\operatorname{dtr}_{k} k\left(v_{2}\right)<d_{2}\left(\operatorname{dtr}_{K} L\right.$ denotes degree of transcendence of $L / K)$. It follows easily that $\operatorname{dtr}_{k} k\left(v_{1}, v_{2}\right)<d_{1}+d_{2}-s$, and in view of our hypothesis on $\operatorname{dim} V$, (i) holds. In turn this implies $\operatorname{dtr}_{k} k\left(v_{2}\right)=d_{2}$, so that $v_{2}$ is a generic point of $Y_{2}$, and (ii) holds. Finally, let $V^{\prime}$ be an irreducible component of $Y_{1} \times{ }_{z} Y_{2}$ containing $V$; replacing $V$ by $V^{\prime}$ above shows that $\operatorname{dim} V^{\prime}=\operatorname{dim} V=d_{1}+d_{2}-s$, whence $\bar{V}=V^{\prime}$, and (iii) holds.

(b) Since $G_{1}$ and $G_{2}$ are dominating, so are the maps $p r_{1}$ and $p r_{2}$ and thus at least one irreducible component of $Y_{1} \times_{z} Y_{2}$ must dominate (the irreducible) $Y_{2}$. Let $W_{1}, \ldots, W_{k}$ be the set of all such components. Now the generic (geometric) fiber $F$ of $p r_{2}$ is irreducible, it being isomorphic to the corresponding irreducible generic fiber of $G_{1}$. Since $F \subseteq \cup_{i=1}^{k} W_{i}$, we have $F \subseteq W_{c}=$ $W$ for some index $c$. On the other hand, a generic point of each $W_{i}$ lies in $F \subseteq W$, hence $W_{i} \subseteq W$, which proves the uniqueness of $W$. To see $\operatorname{dim} W=$ $d_{1}+d_{2}-s$, note that $W$ dominates $Y_{2}$ (of dimension $d_{2}$ ) with generic fiber $F$ of dimension $d_{1}-s$. 
Theorems 1 and 2 describe the fibers of the mapping $f_{\pi}: X(\pi) \rightarrow A$, given hypotheses on the partition $\pi$. A key idea in the proof of both theorems is to interpret the fiber $f_{\pi}^{-1}(a)(a \in A)$ as a fiber product of two smaller varieties (and then to apply Lemma 3) as follows: Break up $\pi=\left(n_{1}, \ldots, n_{r}\right)$ into subpartitions $\pi_{1}=\left(n_{1}, \ldots, n_{s}\right), \pi_{2}=\left(n_{s+1}, \ldots, n_{r}\right)$, and form the diagram with cartesian square:

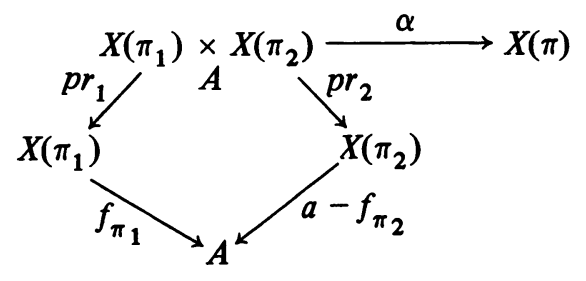

where $a-f_{\pi_{2}}$ is the map given by composing $f_{\pi_{2}}$ with inversion and then translation by $a$, and $\alpha$ is the map that "adds 0-cycles." One checks easily that the image of $\alpha$ in $X(\pi)$ is the fiber $f_{\pi}^{-1}(a)$.

TheOREM 1. Let $m_{0}$ be the least $m>0$ such that $f_{\pi}: X(\pi) \rightarrow A$ is surjective whenever size $(\pi)>m$. Then for any partition $\pi$ of size $r>m_{0}+q$, every fiber of $f_{\pi}$ is equidimensional of dimension $d r-q$, where $d=\operatorname{dim} X, q=\operatorname{dim} A$.

Remark. According to Proposition $2, m_{0}<q-\operatorname{dim} f(X)+1$.

Proof. Let $a$ be a point of $A$ and let $U$ be an irreducible component of the fiber $f_{\pi}^{-1}(a)$. By extending the ground field, we may assume that $X, A$ and $U$ are all defined over the algebraically closed ground field $K$, and that $a$ is $K$-rational. We have that $\operatorname{dim} U>d r-q$ by the theorem on the dimension of fibers of a morphism [13, p. 60]. We must prove this inequality is an equality.

Let $u=\left(\sum_{i=1}^{r} n_{i} x_{i}\right)$ be a generic point of $U$, where $\pi=\left(n_{1}, \ldots, n_{r}\right)$, as in (3). We first claim that

(9) after renumbering the $x_{i}$ (and $n_{i}$ accordingly), $\left\{x_{1}, \ldots, x_{r-q}\right\}$ is a set of independent generic points of $X$ over $K$.

To prove this, note that the map $\theta_{\pi}: X^{r} \rightarrow X(\pi)$ which defined $X(\pi)$ above (1) induces an inclusion of residue fields

$$
K(u) \rightarrow K\left(x_{1}, \ldots, x_{r}\right),
$$

since $\theta_{\pi}\left(\left(x_{1}, \ldots, x_{r}\right)\right)=\left(\sum_{i=1}^{r} n_{i} x_{i}\right)=u$. Since $K(u)$ is the function field of $U$, we see that

$$
\operatorname{dtr}_{K} K\left(x_{1}, \ldots, x_{r}\right)>\operatorname{dtr}_{K} K(u)=\operatorname{dim} U>d r-q .
$$

But $K\left(x_{1}, \ldots, x_{r}\right)$ is the top of the tower

$$
K \subseteq K\left(x_{1}\right) \subseteq K\left(x_{1}, x_{2}\right) \subseteq \cdots \subseteq K\left(x_{1}, \ldots, x_{r}\right),
$$

and since $\operatorname{dtr}_{K} K\left(x_{i}\right) \leqslant d$ for all $i$, at least $r-q$ of the steps in the tower must 
raise the $\mathrm{dtr}$ by the amount $d$, otherwise $\operatorname{dtr}_{K} K\left(x_{1}, \ldots, x_{r}\right)<d r-q$. Renumbering the $x_{i}$, we may suppose these to be the first $r-q$ steps, and we obtain $\operatorname{dtr}_{K} K\left(x_{1}, \ldots, x_{r-q}\right)=d(r-q)$, which is equivalent to (9).

Assuming this renumbering accomplished, break up $\pi$ as follows: $\pi=$ $\left(\pi_{1}, \pi_{2}\right)$, with $\pi_{1}=\left(n_{1}, \ldots, n_{r-q}\right)$ and $\pi_{2}=\left(n_{r-q+1}, \ldots, n_{r}\right)$, and form the diagram (8). Let $v_{1}=\left(\sum_{i=1}^{r-q} n_{i} x_{i}\right)$ and $v_{2}=\left(\sum_{i=r-q+1}^{r} n_{i} x_{i}\right)$, and let $V$ be the closed irreducible subvariety of $X\left(\pi_{1}\right) \times{ }_{A} X\left(\pi_{2}\right)$ having generic point $\left(v_{1}, v_{2}\right)$. Clearly $V$ dominates $U$ under the mapping $\alpha$ (since $\alpha\left(v_{1}, v_{2}\right)=u$ ), so $\operatorname{dim} V$ $>\operatorname{dim} U>d r-q$. We now show, using (a) of Lemma 3, that $\operatorname{dim} V=d r-$ $q$, which completes the proof. The last inequality already checks the hypothesis required on $\operatorname{dim} V$; it remains to check that $V$ dominates $X\left(\pi_{1}\right)$ and that $X\left(\pi_{1}\right)$ dominates $A$. But by construction $v_{1}=\left(\sum_{i=1}^{r-q} n_{i} x_{i}\right)$ is a generic point (2) of $X\left(\pi_{1}\right)$, so $V$ dominates $X\left(\pi_{1}\right)$, and $X\left(\pi_{1}\right)$ dominates $A$ since $\pi_{1}$ has size $r-q>m_{0}$. Now Lemma 3, part (a), (i) shows that $\operatorname{dim} V=d r-q$, and Theorem 1 is proved.

Let $r_{0}$ be the least $r>0$ such that for any partition $\pi$ of size $>r$, the map $f_{\pi}$ is surjective and has all fibers equidimensional of dimension $d r-q$. Then by Theorem 1,

(10) $r_{0}$ exists and satisfies $r_{0} \leqslant 2 q-\operatorname{dim} f(X)+1$.

Proposition 3. If $n>r_{0}$, then every irreducible component of every fiber of $f_{n}: X(n) \rightarrow A$ meets the smooth locus of $X(n)$.

Proof. Let $U$ be a component of $f_{n}^{-1}(a)$. If $U$ contains no smooth points of $X(n)$ (points of size $n$ ), then clearly $U \subseteq X(\pi) \cap f_{n}^{-1}(a)=f_{\pi}^{-1}(a)$, where $\pi=(1,1, \ldots, 1,2)$ is the partition of weight $n$ and size $n-1$. Since $n>r_{0}$, $\operatorname{dim} U=d n-q$. On the other hand, since $n-1>r_{0}, f_{\pi}^{-1}(a)$ has dimension $d(n-1)-q$, contradicting $U \subseteq f_{\pi}^{-1}(a)$.

THEOREM 2. Suppose that $f_{m}: X(m) \rightarrow A$ is surjective and has irreducible generic fiber. Let $\pi$ be a partition of size $r$ for which at least $m+q$ of the parts equal 1. Then every fiber of $f_{\pi}: X(\pi) \rightarrow A$ is irreducible and of dimension $d r-q$.

Remarks. For the partition $(1, \ldots, 1)$ of weight and size $n>m+q$, Theorem 2 is just (7). According to (6), the hypothesis on $f_{m}$ is verified for $m>g$, where $g$ is the genus of a generic curve of $X$.

Proof. Let $a$ be a point of $A$ and let $U$ be an irreducible component of the fiber $f_{\pi}^{-1}(a)$. We may assume that $X, A$, and $U$ are all defined over the same algebraically closed ground field $K$ and that $a$ is $K$-rational. We have that $\operatorname{dim} U \geqslant d r-q$ by the theorem on the dimension of fibers.

Write $\pi=\left(n_{1}, \ldots, n_{r}\right)$ and let $u=\left(\sum_{i=1}^{r} n_{i} x_{i}\right)$ be a generic point of $U$. Call $n_{i}$ the associated part of $x_{i}$. We claim that we can choose a subset of $m$ of the 
$x_{i}$, all having associated part 1 , to form a set of independent generic points of $X$ over $K$.

First note that, according to (9), we can choose from among the $x_{i}$ a set $S=\left\{x_{i}, \ldots, x_{i_{r-q}}\right\}$ of independent generic points of $X$ over $K$. Now at least $m$ of the $x_{i} \in S$ must have associated part 1 , since by hypothesis at least $m+q$ of the $x_{i}$ have associated part 1 , and only $q$ of the $x_{i}$ are not in $S$. By renumbering the $x_{i}$ and $n_{i}$, therefore, we may assume that $\left(x_{1}, \ldots, x_{m}\right)$ is a set of independent generic points with associated parts $n_{1}=n_{2}=\cdots=n_{m}$ $=1$.

Now break up $\pi=\left(\pi_{1}, \pi_{2}\right)$ with $\pi_{1}=(1,1, \ldots 1)$ (m parts), $\pi_{2}=$ $\left(n_{m+1}, \ldots n_{\mathrm{r}}\right)$, and form the diagram (8). Let $v_{1}=\left(\sum_{i=1}^{m} n_{i} x_{i}\right)$ and $v_{2}=$ $\left(\sum_{i=m+1}^{r} n_{i} x_{i}\right)$, and let $V$ be the closed irreducible subvariety of $X\left(\pi_{1}\right)$ $\times_{A} X\left(\pi_{2}\right)$ having generic point $\left(v_{1}, v_{2}\right)$. Arguing as in the proof of Theorem 1, we see that $V$ covers $U$ under $\alpha$ and that the hypotheses of Lemma 3, part (a), are satisfied; consequently:

(i) $\operatorname{dim} V=d r-q(\Rightarrow \operatorname{dim} U=d r-q)$,

(ii) $V$ dominates $X\left(\pi_{2}\right)$, and

(iii) $V$ is an irreducible component of $X\left(\pi_{1}\right) \times{ }_{A} X\left(\pi_{2}\right)$.

On the other hand, the hypotheses of Lemma 3, part (b) are also satisfied for (8), namely: $f_{\pi_{1}}=f_{m}$ is surjective and has irreducible generic fiber by hypothesis, and since $f_{\pi_{2}}$ is surjective (size $\left(\pi_{2}\right)=r-m>q$ ), so also is $a-f_{\pi_{2}}$. Thus there is a unique irreducible component $W$ of $X\left(\pi_{1}\right) \times{ }_{A} X\left(\pi_{2}\right)$ dominating $X\left(\pi_{2}\right)$. By (ii) and (iii) above, $V=W$; thus $W$ covers $U$ under $\alpha$. If $U^{\prime}$ were another component of $f_{\pi}^{-1}(a)$, we could repeat the above argument for $U^{\prime}$ and thereby conclude that $W$ covers $U^{\prime}$, whence $U=U^{\prime}$. Thus $f_{\pi}^{-1}(a)$ is irreducible (and of dimension $d r-q$ ), and Theorem 2 is proved.

EXAMPLE. Let $X=C$, a curve of genus $g>1$, and let $J$ be its Jacobian. Let $n=c r$ with $r \geqslant g, c>1$, and let $\pi=(c, \ldots, c)$ ( $r$ parts), a partition of weight $n$ and size $r$. (Thus $\pi$ does not satisfy the hypothesis of Theorem 2.) Let $i: C(r) \rightarrow C(\pi)$ be the map sending $\left(x_{1}+\cdots+x_{r}\right)$ to $\left(c x_{1}\right.$ $\left.+\cdots+c x_{r}\right)$, evidently a bijective morphism. Consider the commutative diagram:

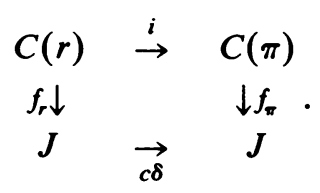

Now the fibers of $f_{r}$ are projective spaces, and the map $c \delta$ is finite to one. Thus the fibers of $f_{\pi}$ are (set theoretically) finite disjoint unions of projective spaces, and in particular are not irreducible.

The end of [10] consists of two examples relevant to statements (6) and (7). 
4. Irreducibility of the fibers of $\varphi_{n}$. We now restrict $X$ to be a (projective, irreducible, and nonsingular) surface, and let $H_{X}^{n}$ be the Hilbert scheme representing 0-dimensional closed subschemes of length $n$ on $X$. As discussed in the introduction, there is a birational map $\sigma_{n}: H_{X}^{n} \rightarrow X(n)$, which is an isomorphism over the smooth locus of $X(n)$, and which desingularizes $X(n)$. We are out to show that for $n \gg 0$, all fibers of the composite mapping

$$
\varphi_{n}: H_{X}^{n} \stackrel{\sigma_{n}}{\rightarrow} X(n) \stackrel{f_{n}}{\rightarrow} A
$$

(which is the Albanese mapping of $H_{X}^{n}$ ) are irreducible and of dimension $2 n-q$; this is a corollary of Theorem 3. For the proof of Theorem 3 we need, in addition to the work above on the morphisms $f_{\pi}: X(\pi) \rightarrow A$, the following fact concerning the behavior of $\sigma_{n}$ over the singular locus:

(11) If $\left(x_{1}+\cdots+x_{n}\right) \in X(n)$ has size $r$, then $\operatorname{dim} \sigma_{n}^{-1}\left(x_{1}+\cdots+x_{n}\right)$ $=n-r[5$, p. 820].

THEOREM 3. Let $X$ be an irreducible, nonsingular, and projective algebraic surface, defined over an algebraically closed field of any characteristic. If $n>r_{0}+q$ (where $r_{0}$ is as in (10)), then

(a) every fiber of the Albanese mapping

$$
\varphi_{n}: H_{X}^{n} \stackrel{\sigma_{n}}{\rightarrow} X(n) \stackrel{f_{n}}{\rightarrow} A
$$

is equidimensional of dimension $2 n-q$, and

(b) for all $a \in A$, the mapping $V \mapsto \sigma_{n}(V)$ is a bijection from the set of irreducible components of $\varphi_{n}^{-1}(a)$ to the set of irreducible components of $f_{n}^{-1}(a)$. Moreover, $V$ and $\sigma_{n}(V)$ are birationally equivalent under $\sigma_{n}$.

Proof. We remark that the hypothesis $n>r_{0}+q$ implies, in particular, that $f_{n}$ and (therefore) $\varphi_{n}$ are surjective.

Let $a$ be any point of $A$, and $V$ an irreducible component of $\varphi_{n}^{-1}(a)$. By the theorem on the dimension of fibers, $\operatorname{dim} V>2 n-q$.

Since $V$ is irreducible, its image $\sigma_{n}(V)$ is an irreducible subvariety of some component $U$ of $f_{n}^{-1}(a)$. We shall prove that $\sigma_{n}(V)=U$.

Let $v$ be a generic point of $V$, and suppose that $\sigma_{n}(v)=\left(\sum_{i=1}^{r} n_{i} x_{i}\right)$ is a point of size $r$, where $r<n$. Then clearly

$$
\sigma_{n}(V) \subseteq X(\pi) \cap f_{n}^{-1}(a)=f_{\pi}^{-1}(a),
$$

where $\pi$ is the partition $\left(n_{1}, \ldots, n_{r}\right)$. We claim that $r>n-q$.

To prove the claim, first note that the theorem on the dimension of fibers applied to $\sigma_{n} \mid V$ shows

$$
\begin{aligned}
2 n-q & <\operatorname{dim} V=\operatorname{dim} \sigma_{n}(V)+\operatorname{dim}\left(\sigma_{n} \mid V\right)^{-1}\left(\sigma_{n}(v)\right) \\
& <\operatorname{dim} \sigma_{n}(V)+n-r,
\end{aligned}
$$

where the second line follows from (11). Now using in (12) the crude estimate 


$$
\operatorname{dim} \sigma_{n}(V)<\operatorname{dim} f_{\pi}^{-1}(a)<\operatorname{dim} X(\pi)=2 r,
$$

we see that

$$
2 n-q<\operatorname{dim} V<2 r+n-r,
$$

thus $n-q<r$, as claimed.

Since $\pi$ has size $r>n-q>r_{0}$, we have that $f_{\pi}^{-1}(a)$ is equidimensional of dimension $2 r-q$. This yields the refined estimate

$$
\operatorname{dim} \sigma_{n}(V)<\operatorname{dim} f_{\pi}^{-1}(a)=2 r-q ;
$$

using this in (12) instead of $2 r$ gives

$$
2 n-q<n+r-q,
$$

which shows that $n<r$, therefore $n=r$, and $\operatorname{dim} \sigma_{n}(v)<2 n-q$. Consequently (again using (12)),

$$
\operatorname{dim} V=\operatorname{dim} \sigma_{n}(V)=2 n-q
$$

which proves (a).

Moreover, every fiber of $f_{n}: X(n) \rightarrow A$ is equidimensional of dimension $2 n-q$ since $n>r_{0}$. Thus $\operatorname{dim} U=2 n-q$, and since $\sigma_{n}(V)$ is an irreducible subvariety of $U$ of dimension $2 n-q$, we conclude that $\sigma_{n}(V)=U$, i.e., $\sigma_{n}(V)$ is an irreducible component of the fiber $f_{n}^{-1}(a)$.

It remains to prove (b), which asserts the bijectivity of the map $V \mapsto \sigma_{n}(V)$ $=U$, and the birationality of $V$ and $U$ under $\sigma_{n}$. But by Proposition 3 (which applies since $n>r_{0}$ ) every component of $f_{n}^{-1}(a)$ meets the open set where $\sigma_{n}$ is an isomorphism; from this (b) follows immediately and Theorem 3 is proved.

COROLlARY 1. If $n \gg 0$ (more precisely: if $n$ is such that the conclusions of Theorem 3 and (7) hold simultaneously), then every fiber of the Albanese mapping

$$
\varphi_{n}: H_{X}^{n} \stackrel{\sigma_{n}}{\rightarrow} X(n) \stackrel{f_{n}}{\rightarrow} A
$$

is irreducible of dimension $2 n-q$, and birationally equivalent to the corresponding fiber of $f_{n}$.

Proof. An immediate deduction from (7) and Theorem 3.

We recall that a variety is called regular if it has trivial Albanese variety. In [8] it is proved that if $V$ is a projective irreducible variety of any dimension, then for $n \gg 0$ every fiber of the map

$$
f_{n}: V(n) \rightarrow \operatorname{Alb}(V)
$$

is a regular variety. Applying this when $V=X$, we obtain

COROllaRy 2. If $n \gg 0$, every fiber of $\varphi_{n}: H_{X}^{n} \rightarrow A$ is a regular variety. 
Proof. Since being a regular variety is a birational invariant, the corollary follows from Corollary 1.

Let $g: V \rightarrow W$ be a dominating morphism of irreducible varieties. We say that a property $P$ holds for the general fiber of $g$ if there is a nonempty open subset of $W$ over which all the fibers have the property $P$. We recall that

(13) the general fiber of $g$ is irreducible if and only if the field extension $k(V) / k(W)$ is primary (i.e., the algebraic closure of $k(W)$ in $k(V)$ is purely inseparable over $k(W))[9$, p. 90].

Since $H_{X}^{n}$ and $X(n)$ are birationally equivalent, the field extensions $k\left(H_{X}^{n}\right) / k(A)$ and $k(X(n)) / k(A)$ are the same. Thus the general fiber of $\varphi_{n}$ is irreducible (and birationally equivalent to the corresponding general fiber of $f_{n}$ ) as soon as the general fiber of $f_{n}$ is irreducible. This is possibly useful in seeking to sharpen the lower bounds on $n$ in the above results, at least in certain cases (e.g. Theorem 4(b) of Part 2). The following example shows what can go wrong at the special fiber.

EXAMPLE. Let $A$ be an abelian surface and let $X$ be the surface obtained by blowing up $A$ at its identity $e$; the general fiber of the Albanese mapping $f$ : $X \rightarrow A$ is thus a point, and $f_{n}^{-1}(e)=D$, a projective line. Since the general fiber of $f_{n}$ is irreducible when $n=1$, all of the fibers of $f_{n}$ are irreducible when $n=1+2=3$, by (7). However, when $n=2, f_{2}^{-1}(e)$ consists of two irreducible components of dimension 2: one component $U_{1}$ is essentially the fiber of the addition map $A(2) \rightarrow A$ over $e$, and the other component $U_{2}$ is $D(2)$. (The general fiber of $f_{2}: X(2) \rightarrow A$ is irreducible.)

There are irreducible components $V_{1}, V_{2}$ of $\varphi_{2}^{-1}(e)$ dominating $U_{1}, U_{2}$ respectively. Since the generic points of $U_{i}$ have size $n=2$, it follows that $\sigma_{2}$ : $V_{i} \rightarrow U_{i}$ is generically $1-1$, hence $\operatorname{dim} V_{i}=2$ for $i=1,2$. But let $L$ be the locus in $U_{2}=D(2)$ of points of size 1 ; clearly $\operatorname{dim} L=1$ and, by (11), $\operatorname{dim} \sigma_{2}^{-1}(x)=1$ for all $x \in L$. Thus there is a two-dimensional locus $V_{3}$ in $\varphi_{2}^{-1}(e)$ dominating $L$, therefore $\varphi_{2}^{-1}(e)$ has at least three components. This shows that (b) of Theorem 3 need not be true for low values of $n$.

\section{PART 2}

In Part 2 we study the special case of the Albanese mapping where $X$ is taken to be an abelian variety $A$ of dimension $q>0$. Then the map $f_{n}$ : $A(n) \rightarrow A$ is given by addition (in $A$ ) of the points of the 0-cycles, i.e., $f_{n}\left(\left(a_{1}+\cdots+a_{n}\right)\right)=\sum_{i=1}^{n} a_{i}$. We study the fibers of $f_{n}$ and of the composite mapping

$$
\varphi_{n}: H_{A}^{n} \stackrel{\sigma_{n}}{\rightarrow} A(n) \stackrel{f_{n}}{\rightarrow} A,
$$

the latter especially (but not exclusively) in case $A$ is an abelian surface. We obtain strengthened versions of some of the results of Part 1 (with less effort), and new information by exploiting the group structure of $A$. 
Henceforth, if we say $v$ is a point of a variety $V$, or write $v \in V$, we mean $v$ is a rational point of $V$ (over the algebraically closed ground field), unless otherwise stated. We write $\mu: A \times A \rightarrow A$ for the group law on $A$, and $\mu_{a}$ : $A \rightarrow A$ for translation by $a \in A$ (i.e., $\mu_{a}(b)=a+b$ ). The Hilbert scheme $H_{A}^{n}$ is abbreviated to $H^{n}$; if $Z$ is a 0 -dimensional closed subscheme of length $n$ on $A$, the associated point of $H^{n}$ is written [ $Z$ ].

5. Dimension, irreducibility and regularity of the fibers. We begin by describing natural actions of $A$ on $H^{n}$ and on $A(n)$ for which the morphism $\sigma_{n}: H^{n} \rightarrow A(n)$ is an $A$-morphism. These actions are key tools for all that follows.

If $Z$ is a 0 -dimensional closed subscheme of length $n$ on $A$, we define its translate $Z_{a}$ by $a \in A$ to be the pullback

$$
\begin{array}{ccc}
Z_{a} & \stackrel{\mu_{-a}^{*}}{\rightarrow} & Z \\
\downarrow & & £ \\
A & \rightarrow & A
\end{array}
$$

Similarly, if $\left(a_{1}+\cdots+a_{n}\right) \in A(n)$, we define its translate by $a$ to be the point $\left(\mu_{a}\left(a_{1}\right)+\cdots+\mu_{a}\left(a_{n}\right)\right)$. The actions we seek are given by the morphisms:

$$
\begin{aligned}
T_{n}^{\prime}: H^{n} \times A & \rightarrow H^{n}, \\
([Z], a) & \mapsto\left[Z_{a}\right], \\
T_{n}: A(n) \times A & \rightarrow A(n), \\
\left(\left(a_{1}+\cdots+a_{n}\right), a\right) & \mapsto\left(\mu_{a}\left(a_{1}\right)+\cdots+\mu_{a}\left(a_{n}\right)\right) ;
\end{aligned}
$$

which are obtained as follows.

$T_{n}$ is the morphism induced (by the universal property of a quotient variety $[12$, pp. 57-60]) by the morphism

$$
\begin{aligned}
A^{n} \times A & \rightarrow A(n), \\
\left(\left(a_{1}, \ldots, a_{n}\right), a\right) & \mapsto\left(\mu_{a}\left(a_{1}\right)+\cdots+\mu_{a}\left(a_{n}\right)\right),
\end{aligned}
$$

which is invariant under the usual action of the symmetric group $\Sigma_{n}$ on the first factor.

To obtain $T_{n}^{\prime}$ we must (by the universal property of the Hilbert Scheme [3]) give a closed subscheme $W_{n}^{\prime}$ of $A \times H^{n} \times A=A \times V$, which is finite and flat over $V$, and has as fiber over $([Z], a) \in V$ the subscheme $Z_{a}$. If $W_{n} \subseteq A$ $\times H^{n}$ is the universal closed subscheme, one checks that the pullback 


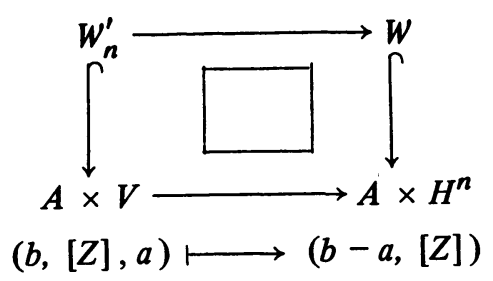

does the job.

REMARKS-NOTATION. Let $H_{a}^{n}$ (resp. $F_{a}^{n}$ ) denote the fibers over $a \in A$ of the mappings $\varphi_{n}$ (resp. $f_{n}$ ), and for all $c \in A$ let $T_{n, c}^{\prime}: H^{n} \rightarrow H^{n}$ (resp. $T_{n, c}$ : $A(n) \rightarrow A(n))$ denote the morphisms of translation by $c$ induced by the actions (14). We remark that translation takes fibers to fibers; more precisely, if $a, b \in A$ and $c$ satisfies $n c=b-a$, then $T_{n, c}^{\prime} \mid H_{a}^{n}: H_{a}^{n} \rightarrow H_{b}^{n}$ and $T_{n, c} \mid F_{a}^{n}$ : $F_{a}^{n} \rightarrow F_{b}^{n}$ are isomorphisms. In this way $A$ acts transitively on the set of fibers of $\varphi_{n}$ (resp. $f_{n}$ ) and the stabilizer of any fiber is the $n$-torsion group of $A$. In particular, all the fibers of $\varphi_{n}$ (resp. $f_{n}$ ) are isomorphic to one another, so anything true for the general fiber or any special fiber is true for all fibers "by translation."

Theorems 4 and 5 below show (among other things) that the conclusions of the corollaries of Theorem 3 hold for all $n>0$, not just for $n \gg 0$, when $X=A$ is an abelian surface.

THEOREM 4. (a) For all $n>0$, each fiber $F_{a}^{n}$ of the mapping $f_{n}: A(n) \rightarrow A$ is irreducible and of dimension $n q-q$, where $q=\operatorname{dim} A$.

(b) If $A$ is an abelian surface, then for all $n>0$, each fiber $H_{a}^{n}$ of the mapping

$$
\varphi_{n}: H^{n} \stackrel{\sigma_{n}}{\rightarrow} A(n) \stackrel{f_{n}}{\rightarrow} A
$$

is irreducible and of dimension $2 n-2$. Furthermore, $H_{a}^{n}$ is birationally equivalent (by restriction of $\sigma_{n}$ ) to the corresponding fiber $F_{a}^{n}$ of $f_{n}$.

Proof. (a) It is enough to prove that the fiber $F_{e}^{n}$ over the identity $e \in A$ is irreducible; the rest follows by translation. But $F_{e}^{n}$ is the image of the composite map

$$
j: A^{n-1} \stackrel{i}{\rightarrow} A^{n} \stackrel{\theta_{n}}{\rightarrow} A(n)
$$

where $i$ is the imbedding sending $\left(a_{1}, \ldots, a_{n-1}\right)$ to $\left(a_{1}, \ldots, a_{n-1},-\sum_{i=1}^{n-1} a_{i}\right)$, and $\theta_{n}$ is the quotient map. Since $A^{n-1}$ is irreducible, so is its image.

(b) Since $A$ is a surface, $H^{n}$ is irreducible (and nonsingular), and $\sigma_{n}$ is a birational map. The argument sketched following (13) shows that (b) holds for the general fiber of $\varphi_{n}$, therefore (b) holds for all fibers by translation. 
TheOREM 5. Let $A$ be an abelian variety of any dimension. Then for all $n>0$, every fiber of the mapping $f_{n}: A(n) \rightarrow A$ is a regular variety (i.e. has trivial Albanese variety).

Proof. By translation, it is enough to prove this for $F_{e}^{n}$, the fiber over the identity. Let $g: F_{e}^{n} \rightarrow B$ be a rational mapping to an abelian variety $B$; we must prove that $g$ is constant.

The surjective morphism $j: A^{n-1} \rightarrow F_{e}^{n}$ given by (15) yields a diagram

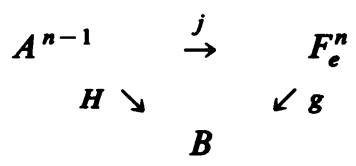

where $H$ is defined by composition, and it is enough to prove that $H$ is constant.

By the general theory of rational maps of abelian varieties (see, e.g. [1]), after a translation of $B, H$ will be a homomorphism of abelian varieties. Since $H\left(\left(a_{1}, \ldots, a_{n-1}\right)\right)$ does not depend on the order of the arguments, all of the homomorphisms

$$
h_{\nu}: A \stackrel{i_{v}}{\rightarrow} A^{n-1} \stackrel{H}{\rightarrow} B,
$$

where $i_{\nu}(1<\nu<n-1)$ is the standard injection to the $\nu$ th factor, are equal. Writing $h$ for any of the $h_{\nu}$, we have

$$
H\left(\left(a_{1}, \ldots, a_{n-1}\right)\right)=\sum_{i=1}^{n-1} h\left(a_{i}\right)
$$

for all $\left(a_{1}, \ldots, a_{n-1}\right) \in A^{n-1}$.

But from (15) it is clear that $\left(a_{1}, \ldots, a_{n-1}\right)$ and $\left(a_{1}, \ldots, a_{n-2},-\sum_{i=1}^{n-1} a_{i}\right)$ have the same image in $F_{a}^{n}$ under $j$, thus

$$
\begin{aligned}
H\left(\left(a_{1}, \ldots, a_{n-1}\right)\right) & =H\left(\left(a_{1}, \ldots, a_{n-2},-\sum_{i=1}^{n-1} a_{i}\right)\right), \text { i.e., } \\
\sum_{i=1}^{n-1} h\left(a_{i}\right) & =\sum_{i=1}^{n-2} h\left(a_{i}\right)-\sum_{i=1}^{n-1} h\left(a_{i}\right)
\end{aligned}
$$

which becomes

$$
\sum_{i=1}^{n-2} h\left(a_{i}\right)+2 h\left(a_{n-1}\right)=e .
$$

Fixing $a_{2}, \ldots, a_{n-1}$ and letting $a_{1}$ vary, we conclude that $h$ and (thus) $H$ are constant, which proves the theorem.

COROLlaRY. Let $A$ be an abelian surface. Then for any $n>0$, every fiber of the map $\varphi_{n}: H^{n} \rightarrow A$ is a regular variety. 
Proof. Being a regular variety is a birational invariant, so the corollary follows from Theorems 4 and 5 .

6. Normality and nonsingularity of the fibers. As remarked earlier, $A$ acts by translation on the fibers of $\varphi_{n}$ and $f_{n}$ transitively but not simply; the stabilizer of any fiber is the $n$-torsion group of $A$. Apparently the fibrations $\varphi_{n}: H^{n} \rightarrow A$ and $f_{n}: A(n) \rightarrow A$ are "twisted products" of the base and a fiber, which one wants to "untwist" into simple products. To do this, form the cartesian diagrams
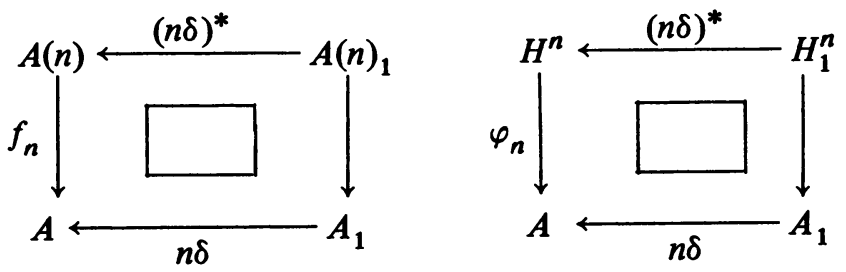

where $A_{1}=A$ and $n \delta$ is the isogeny given by multiplication by $n$. We now show that the fibrations of $A(n)_{1}$ and $H_{1}^{n}$ over $A_{1}$ are actually products.

THEOREM 6. There exist $A_{1}$-isomorphisms

$$
\lambda_{n}^{\prime}: H_{1}^{n} \rightarrow H_{e}^{n} \times A_{1}, \quad \lambda_{n}: A(n)_{1} \rightarrow F_{e}^{n} \times A_{1},
$$

where $e$ is the identity of $A=A_{1}$.

Proof. We write down $\lambda_{n}^{\prime}$ and its inverse using the action $T_{n}^{\prime}$ (14); one obtains $\lambda_{n}$ and its inverse analogously. We note that as point sets,

$$
H_{1}^{n}=\left\{([Z], a) \mid \varphi_{n}([Z])=n a\right\},
$$

and

$$
H_{e}^{n} \times A_{1}=\left\{([Z], a) \mid \varphi_{n}([Z])=e\right\} .
$$

Let $\lambda_{n}^{\prime}$ be the composite morphism

$$
\lambda_{n}^{\prime}: H_{1}^{n} \hookrightarrow H^{n} \times A_{1} \stackrel{1 \times-\delta}{\rightarrow} H^{n} \times A_{1} \stackrel{\left(T_{n}^{\prime}, p r_{2}\right)}{\rightarrow} H^{n} \times A_{1} \stackrel{1 \times-\delta}{\rightarrow} H^{n} \times A_{1}
$$

which on points satisfies

$$
\lambda_{n}^{\prime}([Z], a)=\left(\left[Z_{-a}\right], a\right) \in H_{e}^{n} \times A_{1} .
$$

The natural candidate for the inverse is the composite morphism

$$
H_{e}^{n} \times A_{1} \hookrightarrow H^{n} \times A_{1} \stackrel{\left(T_{n}^{\prime}, p r_{2}\right)}{\rightarrow} H^{n} \times A_{1},
$$

which on points satisfies $([Z], a) \mapsto\left(\left[Z_{a}\right], a\right) \in H_{1}^{n}$.

One checks easily that the composition of these morphisms in either order is the identity, so the theorem is proved. 
In order to exploit Theorem 6, we need the following two facts. Let $V$ and $W$ be irreducible varieties defined over an algebraically closed field.

(17) The product $V \times W$ is normal (resp. nonsingular) if and only if $V$ and $W$ are each normal (resp. nonsingular).

(18) If $g: V \rightarrow W$ is an etale map, then $W$ normal (resp. nonsingular) implies $V$ normal (resp. nonsingular). (The converse holds if $g$ is surjective.)

Proof. (17) If $V$ and $W$ are normal, then so is $V \times W$, by [9, p. 148]. Conversely, let $V^{\prime}$ and $W^{\prime}$ be the normalizations of $V$ and $W$, and consider the finite and birational map $V^{\prime} \times W^{\prime} \rightarrow V \times W$ defined in the obvious way. Since $V \times W$ is normal, Zariski's Main Theorem implies this map is bijective, and hence (ZMT again) an isomorphism, whence $V=V^{\prime}, W=W^{\prime}$. Statement (17) for "nonsingular" is proved in [9, p. 199] (and is not difficult).

(18) These assertions are proved in [4, Exp. 1, 9.2, 9.10].

Let $p$ be the characteristic of the ground field. It is well known that the isogeny $n \delta$ is etale if and only if $p \nmid n[11, p p .64,74]$. Since the pullback of an etale morphism is etale, we have that the morphisms $(n \delta)^{*}$ of $(16)$ are etale if $p \nmid n$. This leads to Corollaries 1 and 2.

Corollary 1. Let $\boldsymbol{A}$ be an abelian surface, and let $\boldsymbol{p} \nmid \boldsymbol{n}>0$. Then every fiber $H_{a}^{n}$ of the map $\varphi_{n}: H^{n} \rightarrow A$ is a nonsingular variety. Moreover, the map $\sigma_{n} \mid H_{a}^{n}: H_{a}^{n} \rightarrow F_{a}^{n}$ is a resolution of the singularities of $F_{a}^{n}$, for all $a \in A$.

Proof. Since $H^{n}$ is nonsingular, the same is true of $H_{1}^{n}$ by (18). But by Theorem $6, H_{1}^{n}$ is isomorphic to the product variety $H_{e}^{n} \times A_{1}$; thus $H_{e}^{n}$ is nonsingular by (17). By translation all of the fibers of $\varphi_{n}$ are nonsingular, and the last statement follows from Theorem 4.

COROllary 2. Let $A$ be an abelian variety of any dimension $q>0$, and let $n>0$ be such that $p \nmid n$. Then every fiber $F_{a}^{n}$ of the map $f_{n}: A(n) \rightarrow A$ is a normal variety.

Proof. Since $A^{n}$ is normal (being nonsingular), the symmetric product is also normal [12, p. 58]. Arguing as in the proof of Corollary 1, we conclude that $A(n)_{1}$ and (hence) $F_{e}^{n}$ are normal; thus all fibers are normal by translation.

REMARK. The normality of the fibers of $f_{n}: A(n) \rightarrow A$ (assured when $p \nmid n$ ) has the consequence that these fibers are themselves quotient varieties for the action of a finite group.

Indeed, let $B$ be the image of the embedding $i: A^{n-1} \rightarrow A^{n}$ used to define the surjective morphism $j: A^{n-1} \rightarrow F_{e}^{n}(15)$. Clearly $B$ is (as set) the kernel of the homomorphism $A^{n} \rightarrow A$ given by addition, and is stable under the action of the symmetric group $\Sigma_{n}$ on $A^{n}$. By restriction $\Sigma_{n}$ acts on $B$, and we may form the quotient variety $B / \Sigma_{n}$. There is a commutative diagram 


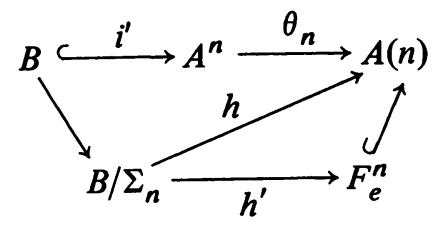

where $h$ is the map resulting from the universal property of the quotient variety, since $\theta_{n} \circ i^{\prime}$ is invariant under the action of $\Sigma_{n}$ on $B$. The map $h$ factors through the image $F_{e}^{n}$ of $\theta_{n} \circ i^{\prime}$ as shown; clearly $h^{\prime}$ is a bijection. We want to prove that $h^{\prime}$ is an isomorphism; this will be so if and only if $h$ is an embedding (closed immersion).

As is pointed out in [12, p. 60], $h$ need not be an embedding in general, unless the characteristic is zero or the group acts freely. However, since $\Sigma_{n}$ does act freely on the open set of off-diagonal points of $A^{n}$ (which $B$ intersects nontrivially), it follows that $h$ is an embedding on an open nonempty set. Consequently $h^{\prime}$ is a bijective birational morphism, and hence an isomorphism (by Zariski's Main Theorem) when $F_{e}^{n}$ is normal. Thus if $p \nmid n$, the fibers of $f_{n}: A(n) \rightarrow A$ are quotient varieties, and the singularities being resolved in Corollary 1 are quotient singularities.

EXAMPLE. Let $A$ be an abelian surface in characteristic 0 , and let $n=2$. Then the map $i: A \rightarrow A^{2}$ sends $a \in A$ to the pair $(a,-a)$, and $B$ is the set of all such pairs (notation as above). Thus $B / \Sigma_{2}=F_{e}^{2}$ is the classical Kummer surface, created by identifying each point $a \in A$ with its inverse $-a$. The singular points of this surface correspond to the points of order 2 in $A$, and are resolved by being blown up into projective lines [14]. This is then a special case of Corollary 1, since the map $\sigma_{2} \mid H_{e}^{2}: H_{e}^{2} \rightarrow F_{e}^{2}$ is an isomorphism except over the singular points of $F_{e}^{2}$, where the fiber is a projective line.

\section{REFERENCES}

1. A. Douady, Variétés abéliennes, Exposé 9 of Sém. C. Chevalley, Variétés de Picard, École Norm. Sup., 1958-59.

2. J. Fogarty, Algebraic families on an algebraic surface, Amer. J. Math. 90 (1968), 511-521.

3. A. Grothendieck, Sém. Bourbaki, Exposé 221, Paris, 1960.

4. A. Grothendieck et al., Revêtements étales et groupe fondamental, (SGA1), Lecture Notes in Math., vol. 224, Springer-Verlag, Berlin and New York, 1971.

5. A. Iarrobino, Punctual Hilbert schemes, Bull. Amer. Math. Soc. 78 (1972), 819-823.

6. Reducibility of the families of 0-dimensional schemes on a variety, Invent. Math. 15 (1972), 72-77.

7. B. Iversen, Linear determinants with applications to the Picard scheme of a family of algebraic curves, Lecture Notes in Math., vol. 174, Springer-Verlag, Berlin and New York, 1970.

8. S. Koizumi, On Albanese varieties, Illinois J. Math. 4 (1960), 358-366.

9. S. Lang, Introduction to algebraic geometry, Interscience Tracts in Pure and Applied Math., no. 5, Interscience, New York, 1958. 
10. A. Mattuck, The irreducibility of the regular series on an algebraic variety, Illinois J. Math. 3 (1959), 145-149.

11. D. Mumford, Abelian varieties, Tata Institute of Fundamental Research Studies in Math., Bombay; Oxford Univ. Press, London, 1970.

12. J. P. Serre, Groupes algébriques et corps de classes, Actualités Sci. Indust., no. 1264, Hermann, Paris, 1959.

13. I. R. Shafarevich, Basic algebraic geometny, Springer-Verlag, Berlin and New York, 1974.

14. E. Spanier, The homology of Kummer manifolds, Proc. Amer. Math. Soc. 7 (1956), 155-160.

Department of Mathiamatics, Sxidmore Collbog, Saratoga Sprnog, New Yorx 12866 